\title{
Global Observations of the 630-nm Nightglow and Patterns of Brightness Measured by ISUAL
}

\author{
Chih-Yu Chiang ${ }^{1, *}$, Tzu-Fang Chang ${ }^{1,2}$, Sunny Wing-Yee Tam ${ }^{2,3}$, Tai-Yin Huang ${ }^{4}$, \\ Alfred Bing-Chih Chen ${ }^{3}$, Han-Tzong $\mathrm{Su}^{1}$, and Rue-Ron $\mathrm{Hsu}^{1}$ \\ ${ }^{1}$ Department of Physics, National Cheng Kung University, Tainan, Taiwan \\ ${ }^{2}$ Plasma and Space Science Center, National Cheng Kung University, Tainan, Taiwan \\ ${ }^{3}$ Institute of Space, Astrophysical and Plasma Sciences, National Cheng Kung University, Tainan, Taiwan \\ ${ }^{4}$ Department of Physics, The Pennsylvania State University Lehigh Valley, Fogelsville, Pennsylvania, USA
}

Received 27 April 2012, accepted 13 December 2012

\begin{abstract}
This study investigates the distributions and occurrence mechanisms of the global local-midnight airglow brightness through FORMOSAT-2/ISUAL satellite imaging observations. We focus on the OI $630.0 \mathrm{~nm}$ nightglow emission at altitudes of $\sim 250 \mathrm{~km}$ along equatorial space. The database used in this study included data from 2007 to 2008 under solar minimum conditions. The data were classified into four specified types in the statistical study. We found that the occurrence of equatorial brightness was often in the vicinity of the geographic equator and mostly at equinoxes with a tendency to move toward the summer hemisphere as the season changes. Conjugate brightness occurring simultaneously on both sides of the geomagnetic equator was observed predominantly in the northern winter. Furthermore, midnight brightness appeared to have lower luminosity from May to July. We suggest that the global midnight brightness associated with the locations and seasons was the result of several effects which include the influence of the thermospheric midnight temperature maximum (MTM), summerto-winter neutral wind, and ionospheric anomalies.
\end{abstract}

Key words: Midnight temperature maximum, Airglow, ISUAL, Ionosphere

Citation: Chiang, C. Y., T. F. Chang, S. W.-Y. Tam, T. Y. Huang, A. B.-C.Chen, H. T. Su, and R. R. Hsu, 2013: Global observations of the 630-nm nightglow and patterns of brightness measured by ISUAL. Terr. Atmos. Ocean. Sci., 24, 283-293, doi: 10.3319/TAO.2012.12.13.01(SEC)

\section{INTRODUCTION}

$\mathrm{OI}\left({ }^{1} \mathrm{D}\right) 630.0 \mathrm{~nm}$ airglow is produced via a charge exchange and the dissociative chemical processes:

$\mathrm{O}^{+}+\mathrm{O}_{2} \rightarrow \mathrm{O}_{2}{ }^{+}+\mathrm{O}$

$\mathrm{O}_{2}^{+}+\mathrm{e}^{-} \rightarrow \mathrm{O}\left({ }^{1} \mathrm{D}\right)+\mathrm{O}$

$\mathrm{O}\left({ }^{1} \mathrm{D}\right) \rightarrow \mathrm{O}\left({ }^{3} \mathrm{P}\right)+\mathrm{h} v(630.0 \mathrm{~nm})$

The $630.0 \mathrm{~nm}$ emission line observed in the F-region ( 200 to $300 \mathrm{~km}$ altitude) is regarded as a sensitive indicator of the electron density based on the $\left[\mathrm{O}^{+}\right] \sim \mathrm{Ne}$ (electron density) approximation (Peterson et al. 1966; Link and Cogger 1988). Therefore, the intensity of the $\mathrm{OI}\left({ }^{1} \mathrm{D}\right) 630.0 \mathrm{~nm}$

\footnotetext{
* Corresponding author

E-mail:johnson@phys.ncku.edu.tw
}

spectrum line is usually used to identify ionospheric density variations, such as the equatorial ionospheric anomaly (EIA) (Rishbeth and Garriot 1969) and traveling ionospheric disturbances (TID) (Saito et al. 2001; Otsuka et al. 2004; Adachi et al. 2011) in the F layer. In particular, enhancement of the $630.0 \mathrm{~nm}$ airglow is typically observed around midnight at equatorial latitudes. The enhancement regions are found to move pole-ward with time. This tendency is described as the midnight brightness waves (MBW) (Colerico et al. 1996), which are the signature of the thermospheric midnight temperature maximum (MTM) effect.

Developing near the geographic equator, the highly variable MTM is a large-scale neutral temperature anomaly with widespread influence on the behavior of nighttime low-latitude thermosphere. It couples with local density to form a pressure bulge and causes two simultaneously poleward moving regions of enhanced OI $630.0 \mathrm{~nm}$ nighttime 
airglow emission, one toward each pole (Herrero and Meriwether 1980; Colerico and Mendillo 2002). Harper (1973) and Spencer et al. (1979) first reported the MTM phenomenon, their cases observed respectively by the incoherent scatter radar from Arecibo and the NATE experiment aboard the Atmospheric Explorer E (AE-E) satellite. The amplitude of the temperature bulge was found to range from $20^{\circ}$ to $200^{\circ} \mathrm{K}$ (Spencer et al. 1979; Burnside et al. 1981; Colerico and Mendillo 2002; Meriwether et al. 2008). In addition, Colerico et al. (1996) reported a pre-midnight brightness wave (PMBW), which typically occurred in the early evening (2000 2200 LT) and moved toward the equator. Colerico and Mendillo (2002) suggested that PMBW may result predominantly from the relaxation of the subtropical arcs (equatorial anomaly) due to reversal of the fountain effect.

Recently, several studies have clarified the general characteristics of latitudinal and seasonal variations of nighttime airglow emissions in the ionosphere using groundbased observations (Otsuka et al. 2003; Niranjan et al. 2006; Shiokawa et al. 2006; Maruyama et al. 2008). However, they could only investigate the ionospheric variations within the localized ground-based observational region and provide a dim macroscopic perspective. In this study, we investigate the features and behavior of $630.0 \mathrm{~nm}$ midnight brightness by analyzing the side-viewing optical images obtained by the Imager of Sprites and Upper Atmospheric Lightning (ISUAL) (Chern et al. 2003), the scientific payload onboard the FORMOSAT-2 satellite. This is the first statistical study of global midnight brightness at low latitudes using satellite observations. In our investigation, the $630.0 \mathrm{~nm}$ midnight brightness is found to feature a seasonal tendency and different longitudinal signatures which require global observation to identify. We propose a possible mechanism of midnight brightness by analyzing the sequence of observations and seasonal variations.

\section{INSTRUMENTATION AND OBSERVATIONS}

The scientific payload ISUAL onboard FORMOSAT-2 satellite contains three bore-sighted sensors: an intensified charge-coupled device (CCD) Imager, a six-channel spectrophotometer (SP) and a 16-anode array photometer (AP). In this study, we focused on the CCD Imager observations with the filter of $630.0 \pm 3.5 \mathrm{~nm}$. The FORMOSAT-2 spacecraft moves along a sun-synchronized orbit at $890 \mathrm{~km}$ altitude, making 14 revolutions per day. When ISUAL tracks from south to north with its eastward configuration, it observes airglow emissions around local midnight ( 2330 LT). The CCD Imager provides a $20^{\circ}$ (horizontal) $\times 5^{\circ}$ (vertical) field-of-view (FOV) with image resolution of $516 \times 128$ pixels.

Figure 1 shows a one-orbit example of ISUAL CCD Imager observations on April 2, 2007. When the ISUAL
CCD Imager tracked from south to north, it took one picture every 30 seconds over an interval of 20 minutes, corresponding roughly to the range of geographic latitudes from $-30^{\circ}$ to $+30^{\circ}$. The exposure time was 1 second throughout the interval. The track of ISUAL footprints and ISUAL FOV ground projections are shown in Fig. 1a. One of the FOV projections is marked and its observational image is highlighted in Fig. 1b. The image reveals two distinct emission layers centered at different altitude levels. The upper emission is due to the $\mathrm{OI}\left({ }^{1} \mathrm{D}\right)$ airglow emission layer and the lower emission is due to the $\mathrm{OH}(9,3)$ airglow emission layer (Burnside et al. 1977; Mende et al. 1993). Rajesh et al. (2009) suggested that most of the $\mathrm{OH}(9,3)$ enhancements observed by ISUAL were the result of the line-of-sight integration of the corresponding $\mathrm{OI}\left({ }^{1} \mathrm{D}\right)$ enhancements. In this study, we focus on the $\mathrm{OI}\left({ }^{1} \mathrm{D}\right)$ enhancements that were the result of plasma density variations. Considering the overlap between adjoining snapshots by ISUAL, we were able to integrate tens of the individual images from a single orbit into a panoramic picture that included a wider latitudinal range to reveal a combined latitudinal-altitudinal distribution of airglow emission covering about $60^{\circ}$ in latitude, Fig. 2 . However, the most important things that needed to be considered before the process of combination were the sensitivity degradation of ISUAL instruments and its impact on observations (Chen et al. 2012). We note that the background increased dramatically at almost 1 count per day for a long exposure time $(1000 \mathrm{~ms})$ and for the higher gain setting, which was typically used to observe nighttime airglow. Moreover, because the satellite view angle has a tilt of a few degrees, the geometry of the Earth's curvature must be calculated in order to align the dimensions of the images with the true horizontal and vertical directions. Then we got the calibrated height definition in the profile. The data used in this study have been treated with the correction processes mentioned above.

\section{ANALYSIS AND RESULTS}

As is well-known, a $630.0 \mathrm{~nm}$ airglow intensity generally varied with geomagnetic activity (Basu et al. 2001; Pimenta et al. 2007). In order to understand the tendency and behavior of midnight brightness, we selected cases from the ISUAL database that took place during a normal quiettime period. The quiet-time cases of nighttime airglow were differentiated from disturbed-time cases based on the $\mathrm{Kp}$ index. When the sum of the $4 \mathrm{Kp}$ values (within 12 hours) before the initiation of an event was in excess of 12, we defined that event as a disturbed-time period and excluded the event from the cases of interest. During the period from February 2007 to October 2008 (under solar minimum conditions), 535 cases of nighttime airglow were identified under a geomagnetically quiet condition and were included in our statistical analysis. 
The integrated ISUAL airglow images, each corresponding to a latitudinal-altitudinal distribution of nighttime airglow emission over a wide range of $\sim 60^{\circ}$ in latitude, enabled us to investigate the nighttime airglow distribution and tendency from a more macroscopic point of view. We classified the tendency and behavior of the midnight brightness into four informative types according to the ISUAL wide-range observational results. The definition of the four classified types is given below along with four examples shown in Fig. 2, each representing a different type. The red arrows at the bottom of the panels indicate the geomagnetic equator and the black arrows indicate the geographic equator. Figure 2a is a case with no brightness during the period of interest; we will use "NB" to abbreviate for this type of cases. Figure $2 b$ shows an example of the cases with the occurrence of single equatorial brightness near the geographic equator; we will use "SEB" to abbreviate for this type of case. Figure $2 \mathrm{c}$ is an example of the cases featuring double equatorial brightness located on different sides of the geographic equator but on the same side with respect to the geomagnetic equator; we will use "DEB" to refer to this pattern of midnight brightness. The case in Fig. 2d, like

(a)

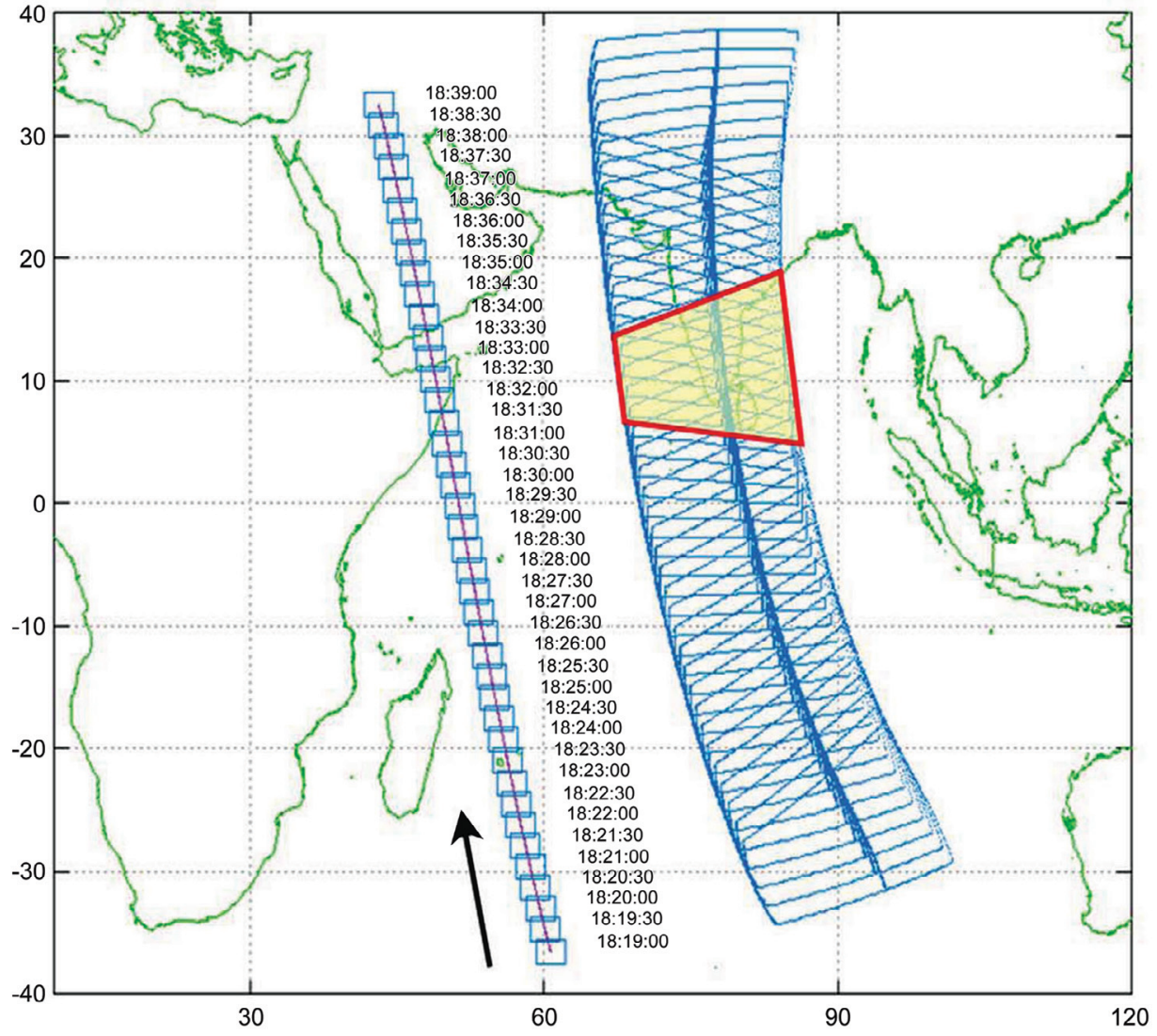

(b)

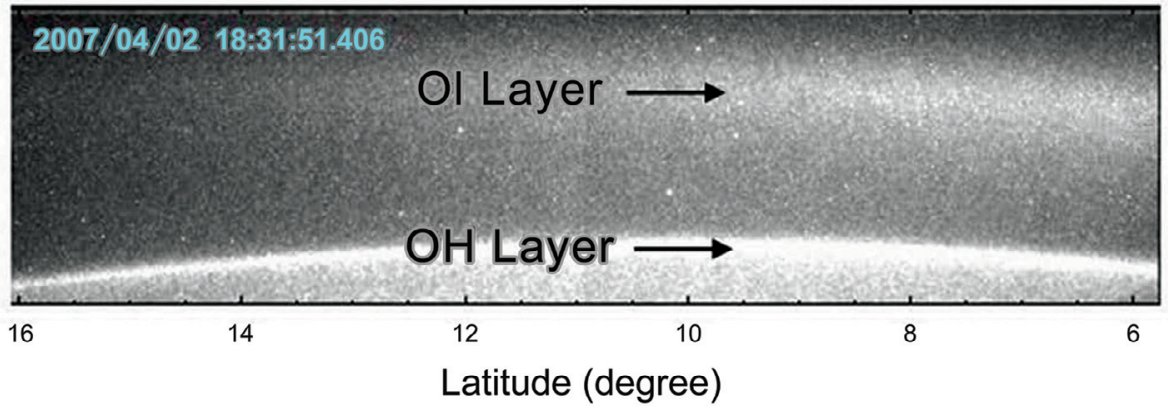

Fig. 1. An example of the ISUAL CCD Imager's one-orbit observation on April 2, 2007. (a) The track of the ISUAL FOV projection and footprint are shown in this figure. During that time, ISUAL took 41 images over 20 minutes and the ISUAL FOVs covered the latitudinal region from $\sim 30^{\circ} \mathrm{S}$ to $\sim 30^{\circ} \mathrm{N}$. One of the FOV projections is marked by red lines and its observational image is shown in (b). (b) This ISUAL observational image of nighttime airglow reveals two emission layers: the upper one is $\mathrm{OI}\left({ }^{1} \mathrm{D}\right)$ airglow emission layer and the lower one is $\mathrm{OH}(9,3)$ airglow emission layer. 
(a) Date: $2008 / 06 / 10 \quad$ Orbit: 02

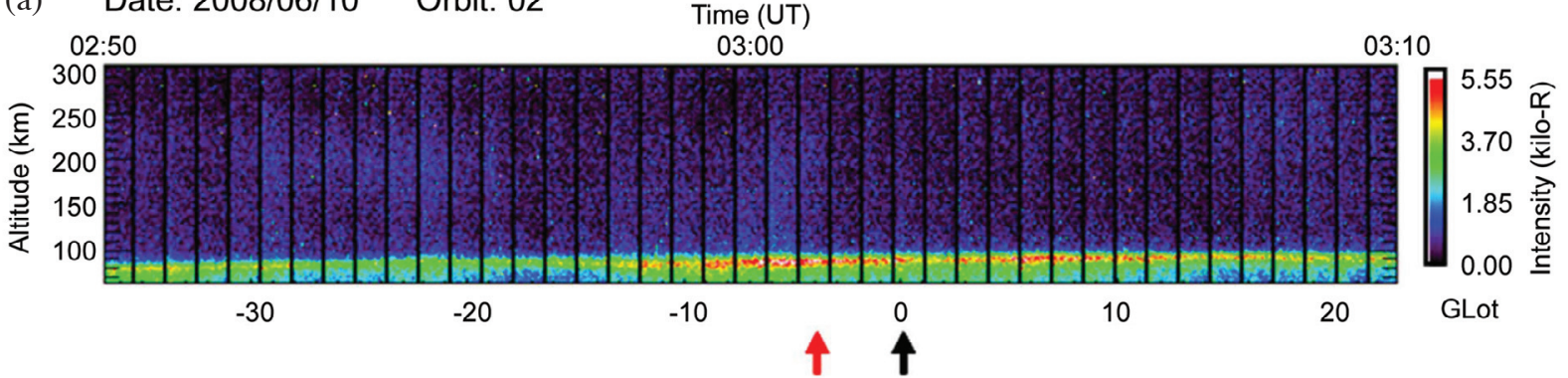

(b) Date: 2008/03/13 Orbit: 05

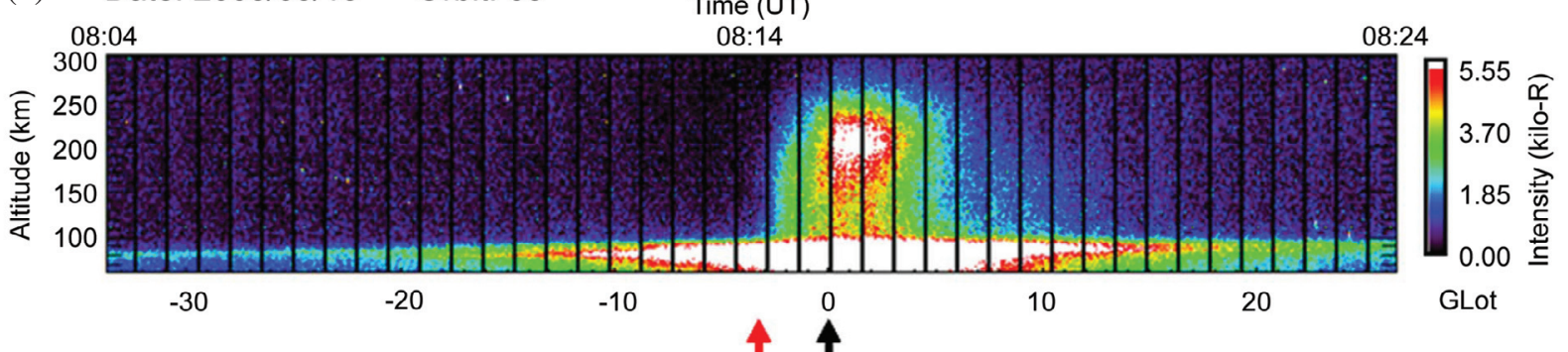

(c) Date: $2008 / 01 / 16 \quad$ Orbit: $14 \quad$ Time (UT)
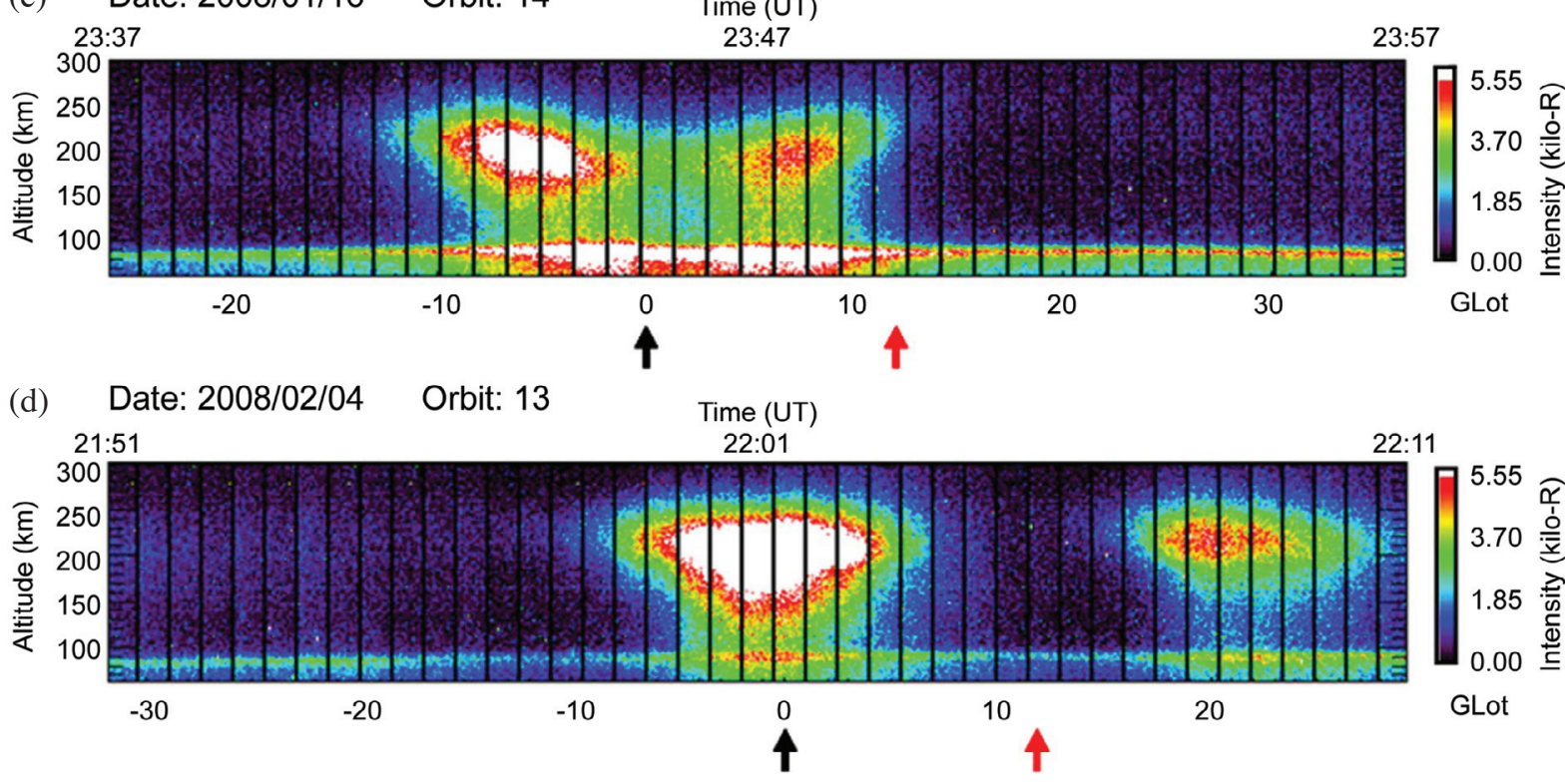

Fig. 2. Four representative cases for the classified types of midnight brightness observed by the ISUAL craft. (a) A case with no brightness (NB), (b) a case with single equatorial brightness (SEB), (c) a case with double equatorial brightness (DEB), and (d) a case with conjugate brightness (CB). The red arrows at the bottom of the panels indicate the geomagnetic equator and the black arrows indicate the geographic equator.

Fig. 2c, features double brightness, but at locations on both sides of the geomagnetic equator; we name this type of case as conjugate brightness with the abbreviation "CB".

In order to further study global variation in different longitudinal regions, we plot the mean $\mathrm{OI}\left({ }^{1} \mathrm{D}\right)$ nightglow intensities at the tangent points of all orbital observations in the global maps in Fig. 3. These distribution maps could provide us the overall morphologies of midnight brightness and allow us to easily find the features among the different longitudinal regions. Orbits (i), (ii) and (iii) were the examples we selected in the map and we will discuss these orbits in detail later. We separated the observation periods to a sequence of seasons: (a) February April 2007; (b) May July 2007; (c) August October 2007; (d) November 2007 $\sim$ January 2008; (e) February April 2008; (f) May June 2008; and (g) August $\sim$ October 2008. There are dashed lines surrounding a region in the southern Pacific Ocean, Fig. 3d, the plot that corresponds to the southern summer (November $2007 \sim$ January 2008). That is a region where observational data were insufficient.

Figure 4 shows the $630.0 \mathrm{~nm}$ nighttime airglow intensity profiles for the longitudinal range of $22 \sim 45^{\circ} \mathrm{E}$ at an 

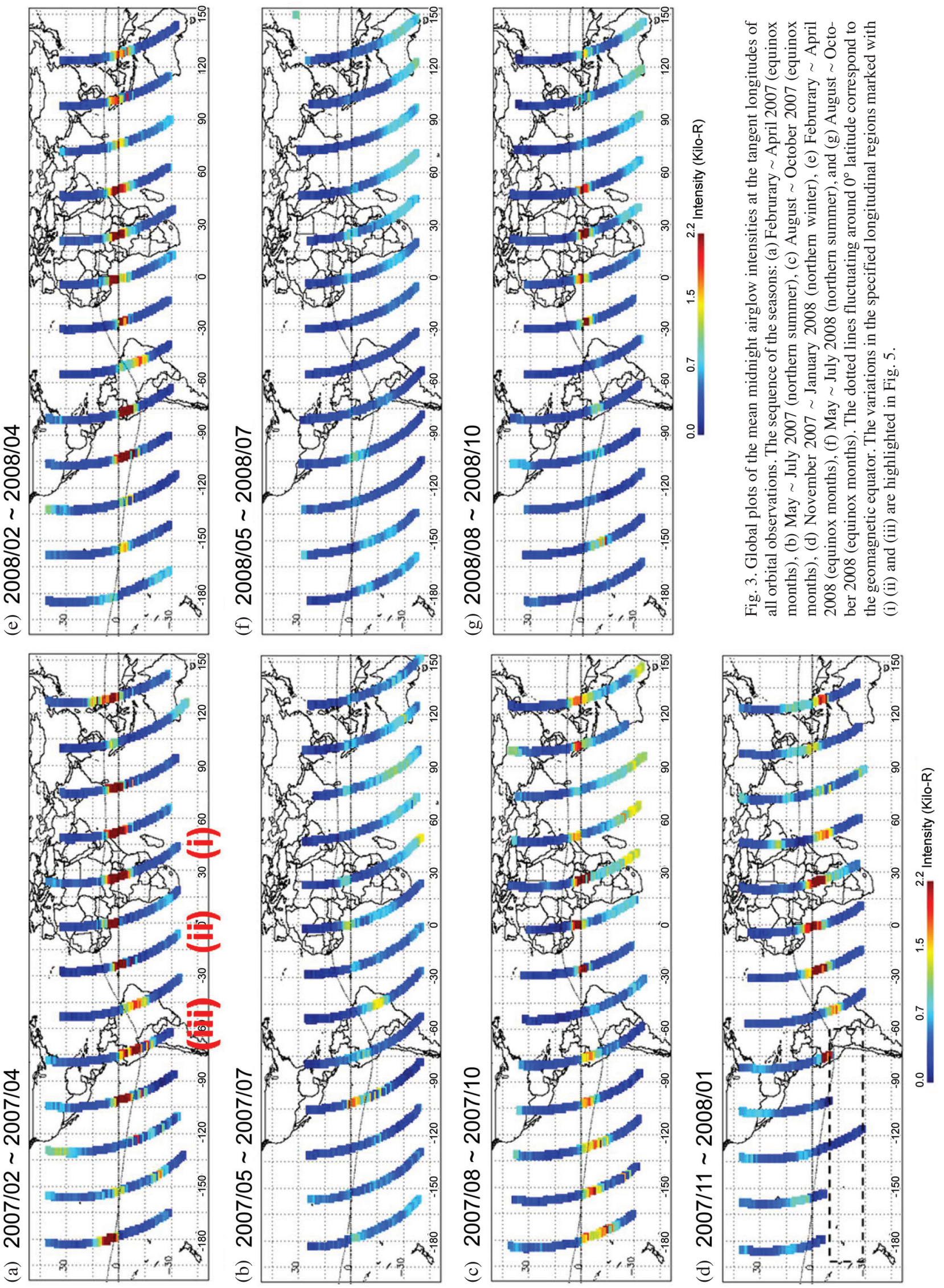

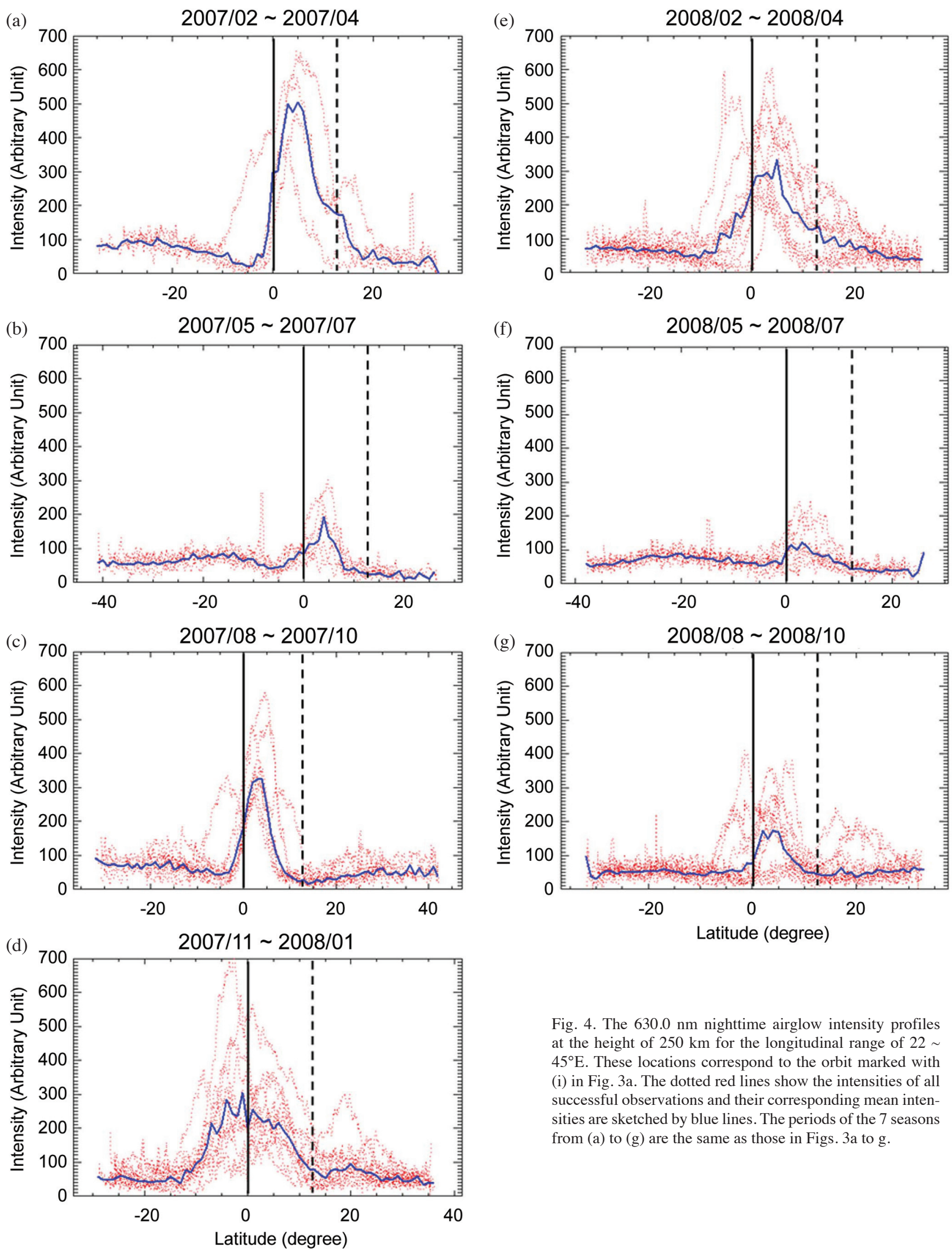

Fig. 4. The $630.0 \mathrm{~nm}$ nighttime airglow intensity profiles at the height of $250 \mathrm{~km}$ for the longitudinal range of 22 $45^{\circ} \mathrm{E}$. These locations correspond to the orbit marked with (i) in Fig. 3a. The dotted red lines show the intensities of all successful observations and their corresponding mean intensities are sketched by blue lines. The periods of the 7 seasons from (a) to (g) are the same as those in Figs. $3 a$ to g. 
altitude of $250 \mathrm{~km}$ around $2300 \mathrm{LT}$ during observation periods [such locations correspond to the orbit marked with (i) in Fig. 3a]. The periods of the different seasons from (a) to $(\mathrm{g})$ are the same as those in Fig. 3. The red dotted lines show the intensities of all successful observations in each season and the blue lines indicate the corresponding mean intensity of the observations. The vertical black dashed lines indicate the geomagnetic equator and the solid lines indicate the geographic equator. In these results, we see that the midnight brightness mostly occurred in the vicinity of the geographic equator. The nighttime airglow was constantly influenced by seasonal effect, featuring midnight brightness of relatively low intensity during the northern summer (May $\sim$ July).

In order to find out whether there is any variation trend of midnight brightness in relation to season and latitude under different properties of the local geomagnetic field, we examine the $\mathrm{OI}\left({ }^{1} \mathrm{D}\right)$ nightglow brightness results (Fig. 5) based on one-month intervals during which the ISUAL instrument was available to provide data that served the pur- pose of this study. We found that the latitudinal locations of midnight brightness showed significant seasonal variations. Figure 5 shows the observations from three different longitudinal regions [(i), (ii) and (iii)] that correspond to the orbits with the same labels in Fig. 3a. These three orbits had different declination angles. Orbit (i) was in the longitudinal region (between $-15^{\circ} \sim+150^{\circ}$ longitude) where the geomagnetic equator is northward of the geographic equator with the declination angle around $0^{\circ}$. Orbit (iii) was in the region (between $-85^{\circ} \sim-60^{\circ}$ longitude) with the geomagnetic equator southward of the geographic equator and the declination angle around $0^{\circ}$. Orbit (ii) was in the geographic region between $-60^{\circ} \sim-15^{\circ}$ longitude, with a declination angle around $-20^{\circ}$ (westward). The solid lines and dashed lines indicate the geographic equator and geomagnetic equator, respectively.

In order to understand the possible mechanisms for the occurrence of the four classified types of midnight brightness, shown in Fig. 2, we examine the occurrence rates of the four brightness types from all the orbits combined.
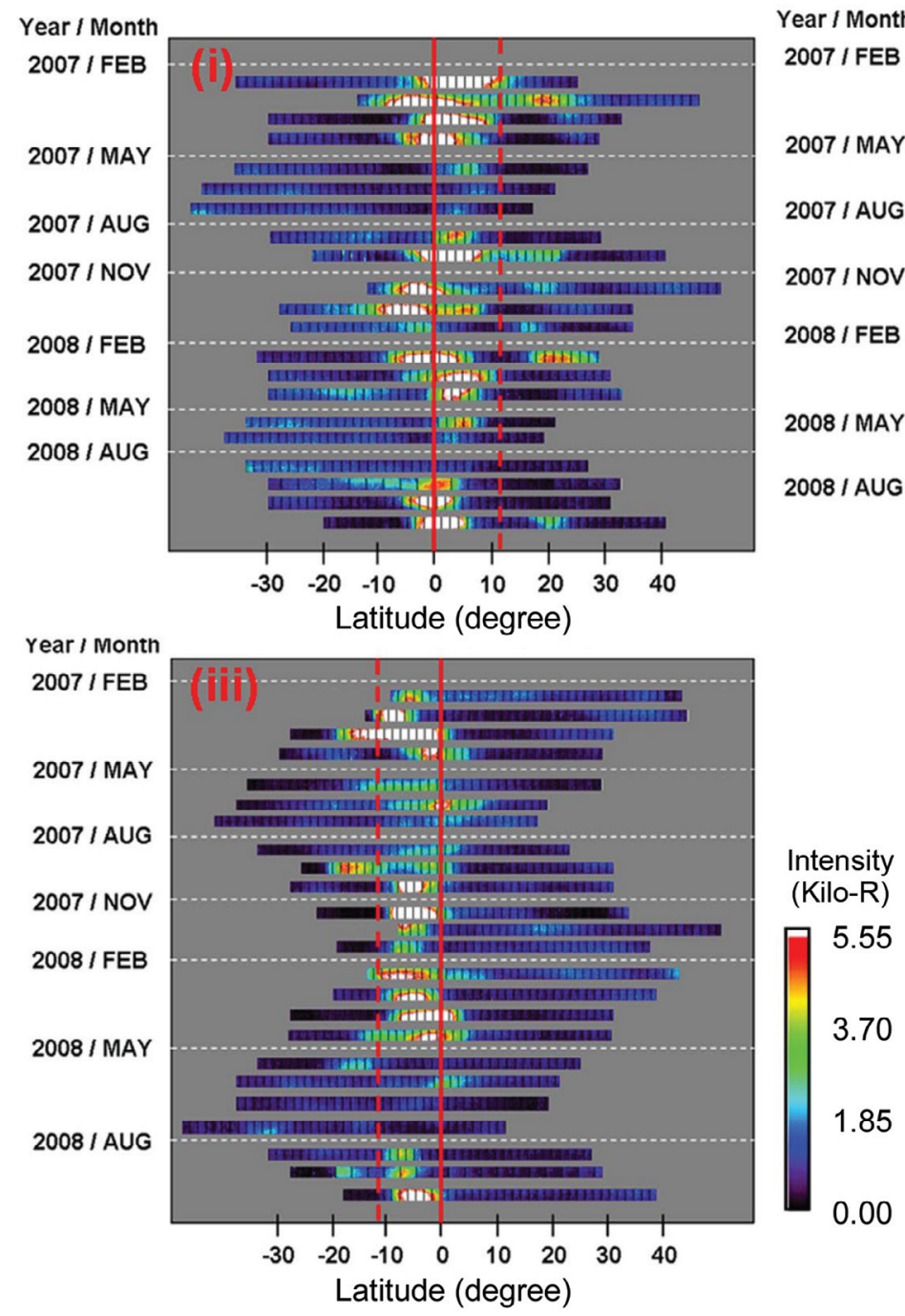

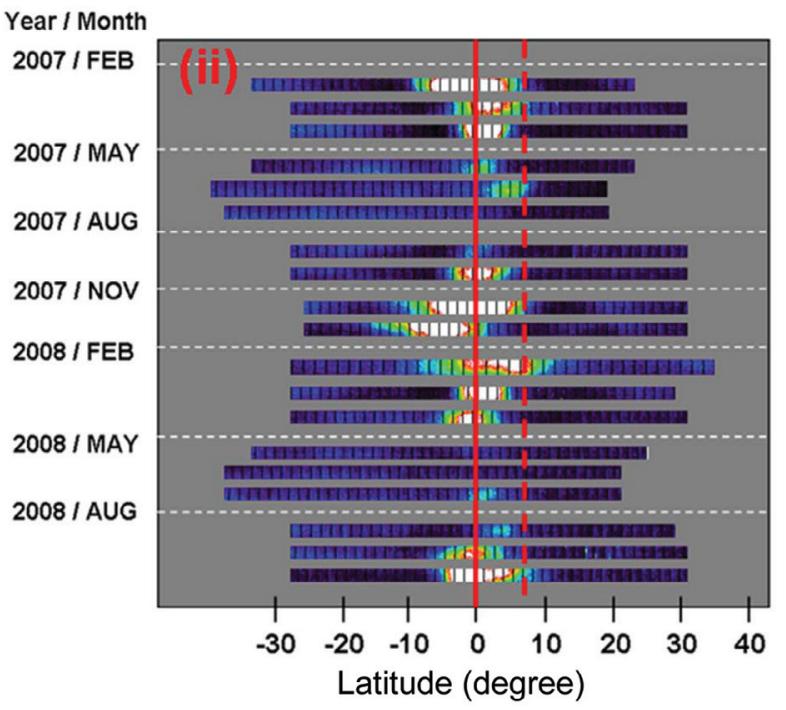

Fig. 5. Sequencing data observed from four different longitudinal regions during the 7 seasons. The regions (i), (ii) and, (iii) correspond to those with the same labels shown in Fig. 3a. The dotted lines indicate the geomagnetic equator; the solid lines indicate the geographic equator. 
These occurrence rates are shown in Fig. 6 with bar plots for each month. The cases with single equatorial brightness (SEB) are in green, double equatorial brightness (DEB) in yellow, conjugate brightness (CB) in red, and no brightness $(\mathrm{NB})$ in blue. The four occurrence rates exhibit clear periodicity. According to our observations, the occurrence rate of SEB peaked near the equinoxes; that of $\mathrm{CB}$ peaked near the northern winter; that of NB peaked near the northern summer; and the occurrence rate of DEB was quite low each month.

\section{DISCUSSION}

The features of the annual anomaly were investigated in previous ionospheric studies. It was found that the F2layer peak electron density (NmF2) at the December solstice was $20 \%$ higher than that at the June solstice (Yonezawa et al. 1959). Also, the total electron content (TEC) around the December solstice exceeded that at the June solstice in both the northern and southern hemispheres (Titheridge et al. 1983). These features of the annual anomaly are demonstrated in our observations as well. From Fig. 5, ISUAL observations found that the equatorial nightglow was significantly dim from May to July, sometimes even without any midnight brightness. The low plasma density around the months of northern summer may also reduce the probability of dynamical processes generating midnight brightness, resulting in generally more cases of NB. So, we suggest that the relatively frequent occurrence of NB in those months (see Fig. 6) is related to the ionospheric annual anomaly.
In this paper, our major goals are to investigate the behaviors of midnight brightness and to consider the possible mechanisms for all kinds of cases. Based upon the early work mentioned in the introduction section, most of the studies on midnight brightness have attempted to associate the effect with in situ temperature and neutral wind measurements (Herrero and Meriwether 1980; Colerico et al. 1996, 2002; Otsuka et al. 2003). Because the brightening moved pole-ward with time from near the geographic equator, forming the $\mathrm{MBW}$, the observation timing was very important. The latitudinal locations of equatorial brightness should depend on the local time.

Adachi et al. (2010) showed the observations based on 14 days of ISUAL data obtained from the Asian sector. The authors noted that the bright airglow emissions were often observed in the equatorial region. Because ISUAL craft primarily observed the region around the local midnight, they suggested that the equatorial airglow consistently found at 0 $\sim 10^{\circ} \mathrm{N}$ probably corresponded to MBW rather than PMBW. Here, we utilize the ISUAL data more comprehensively by considering longer time periods and seasonal variations in order to provide observational evidence of global distributions and seasonal tendencies of midnight brightness.

In our results shown in Fig. 5, we found that the equatorial brightness usually occurred around the geographic equator and slightly toward the geomagnetic equator under the three different magnetic conditions. As documented, the $630.0 \mathrm{~nm}$ emission observed at around an altitude of $250 \mathrm{~km}$ and is regarded as a sensitive indicator of the electron density. We can presume that the MBW moves pole-ward from

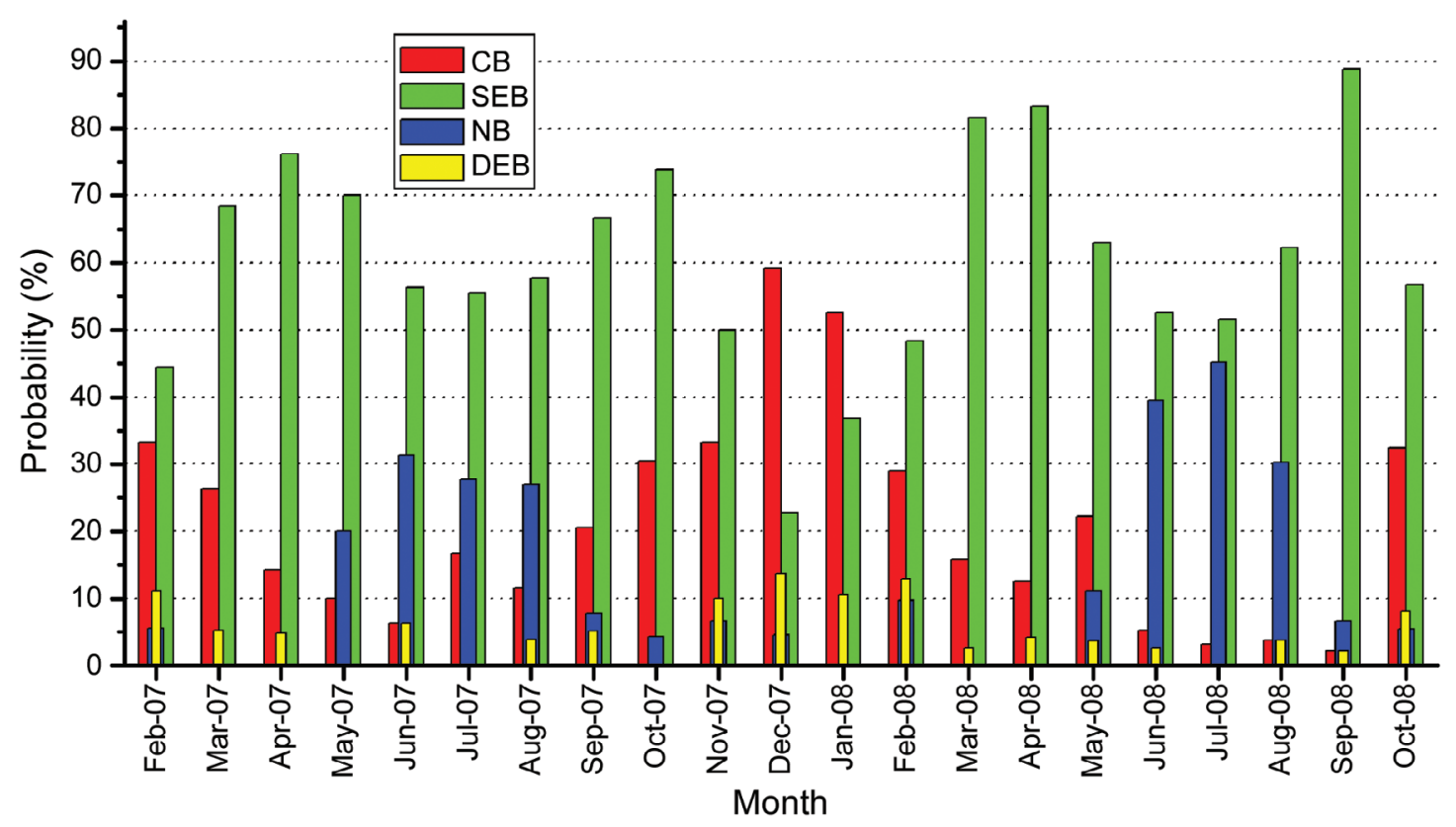

Fig. 6. Combined occurrence rates of the four brightness types from all the orbits in each month. The single equatorial brightness (SEB) cases are in green, double equatorial brightness (DEB) in yellow, conjugate brightness (CB) in red, and no brightness (NB) in blue. 
the geographic equator along the magnetic field lines. Because, in principle, the altitude for a given field line is at its maximum at the geomagnetic equator; the plasma that moves toward the geomagnetic equator would travel upward and the plasma that moves away would go downward. As the plasma is subject to various kinds of recombination, which generally are at higher rates at lower altitudes, the density of the downward moving plasma would soon be reduced significantly. Among the various recombination processes, that consist of reactions (1) to (3) would produce the $630.0 \mathrm{~nm}$ emission. However, there would be other recombination processes that are irrelevant to this emission while reducing the plasma density. The upward moving plasma, on the other hand, travels to altitudes where recombination rates are lower. Thus, their density would be able to be sustained for a longer period allowing the plasma to produce the emission even after the emission from their downward counterpart was exhausted. In other words, if the initial MTM had been formed sufficiently long enough, one would expect to see the emission mainly from the longer-lasting upward moving plasma at the lower magnetic latitudes corresponding to the locations between the geographic and geomagnetic equators. This suggests that the latitudinal locations of equatorial brightness are magnetic field dependent near the equatorial regions. The most notable feature of the structures reported here is the midnight brightness appearing to have a tendency to move toward the summer hemisphere as the season changes. We suggest that this tendency may be associated with the sub-solar point. This is the first report which shows the complete trace of midnight brightness following the variation in season for longitudinal regions characterized with various geomagnetic conditions. The swaying trend of the equatorial brightness was consistent with the signature of MTM seasonal variation reported by Herrero and Spencer (1982). They showed a definite seasonal variation of MTM with summer hemisphere maximum occurring earlier than the winter hemisphere maximum.

Adachi et al. (2010) also mentioned that DEB might represent the cross section of two branches of a V-shaped MBW which had been found in the experimental studies by Meriwether et al. (2008). The ISUAL observation periods were around $2330 \mathrm{LT}$ which was during the typical occurrence periods of MBW, between $2300 \sim 0100 \mathrm{LT}$, when the enhanced brightness propagates pole-ward (Colerico et al. 1996). Thus, the timing of the ISUAL observations is after the branch point of V-shape MBW propagation. According to our results in Fig. 6, the occurrence rate of DEB was quite low every month (less than $15 \%$ ). The low occurrence rate of DEB did not imply that MBW was just an occasional phenomenon because, as discussed, the lifetime of the poleward propagating equatorial brightness could be quite different in the two hemispheres as a result of the inclination of the geomagnetic field lines. Thus, even with MBW, it was possible that only one single core of brightness occurred at a given local time. Also, if the observation time happened to correspond to the branch point of the MBW, one would also find the brightness as one single core. Therefore, most of the events of equatorial brightness were found to belong to the SEB case, as indicated in Fig. 6.

From the occurrence rate of the CB shown in Fig. 6, we note that the $\mathrm{CB}$ occurred relatively more often around northern winter (October February). In earlier studies, Rishbeth and Setty (1961) found that NmF2 was higher in winter than in summer, and they first suggested the possibility of composition change was the cause of the winter anomaly. Torr and Torr (1973) and Rishbeth (1972) suggested that the anomaly might be due to transequatorial neutral wind blowing from the summer hemisphere to the winter hemisphere. From the global maps in Fig. 3, the orbits between $-15^{\circ} \sim+150^{\circ}$ longitudes have the geomagnetic equator located northward of the geographic equator. These orbits constitute a majority among all of the orbits that are taken into consideration in the statistics shown in Fig. 6. Therefore, we usually find the brightness appearing on the other side of the geomagnetic equator during the northern winter and means that the $\mathrm{CB}$ cases are consistent with the winter anomaly. In other words, the $\mathrm{CB}$ might be the results of plasma enhancement induced by the neutral wind effect. In the example of the CB shown in Fig. 2d, there is a region of high emission intensity over the geographic equator accompanying a secondary brightness region on the other side of the geomagnetic equator. We suggest that the occurrence of the secondary brightness region, which made this a CB case, might be caused by the meridional neutral wind from the southern hemisphere. We note that the primary brightness region might be related to the MTM effect which could generate a pressure bulge at midnight geographic equator. The pressure bulge could significantly modify the meridional neutral winds resulting in an abatement of the neutral wind or perhaps even cause a reversal of the overall wind direction in the summer hemisphere (Niranjan et al. 2006).

From the discussion above, we suggest that SEB and DEB are in relation to the MTM effect. We can see that most of the midnight brightness events are equatorial brightness (SEB and DEB). According to our results in Fig. 6, the occurrence rate of SEB peaked around the equinoxes. This occurrence rate is statistically influenced by the relatively frequent occurrence of NB during the northern summer due to the annual anomaly, and the higher occurrence rate of $\mathrm{CB}$ during the northern winter due to the winter anomaly.

\section{CONCLUSION}

We have showed and discussed the seasonal and global tendencies of $\mathrm{OI}\left({ }^{1} \mathrm{D}\right)$ nightglow at low latitudes during the geomagnetically quiet periods. Due to the recent improvement in nightglow observational technology and the availability of the FORMOSAT-2 ISUAL data, this is, as far as 
we know, the first statistical report that discusses the global midnight brightness. We found that midnight brightness was controlled by different sources at different locations. First, NB was associated with the ionospheric annual anomaly during May to July. Second, we suppose that SEB and DEB were associated primarily with the MTM effect and the featured temperature variation. Third, the CB case, however, was associated largely with the winter anomaly which the neutral wind plays a role in its formation. It is necessary to take into account the locations and seasons when explaining the mechanism of midnight brightness occurrence. Overall, the global midnight brightness can be contributed by several effects including the influence of the MTM effect, summerto-winter neutral wind and ionospheric anomaly.

Acknowledgements The authors acknowledge the Taiwan National Space Organization (NSPO) and FORMOSAT-2/ ISUAL science and operator team. The work by Chih-Yu Chiang and Sunny Wing-Yee Tam was supported by National Science Council (NSC) Grant NSC 99-2111-M-006002-MY3 to National Cheng Kung University (NCKU). TaiYin Huang acknowledges support from the US NSF Grant AGS-0836920 to The Pennsylvania State University and Taiwan's NSC Grant NSC 98-2811-M-006-018 to NCKU. Alfred Bing-Chih Chen, Han-Tzong Su, and Rue-Ron Hsu acknowledge support from the NSPO-S-100010 to NCKU.

\section{REFERENCES}

Adachi, T., M. Yamaoka, M. Yamamoto, Y. Otsuka, H. Liu, C. C. Hsiao, A. B. Chen, and R. R. Hsu, 2010: Midnight latitude-altitude distribution of $630 \mathrm{~nm}$ airglow in the Asian sector measured with FORMOSAT-2/ISUAL. J. Geophys. Res., 115, A09315, doi: 10.1029/20 09JA015147. [Link]

Adachi, T., Y. Otsuka, M. Yamaoka, M. Yamamoto, K. Shiokawa, A. B. Chen, and R. R. Hsu, 2011: First satellite-imaging observation of medium-scale traveling ionospheric disturbances by FORMOSAT-2/ISUAL. Geophys. Res. Lett., 38, L04101, doi: 10.1029/20 10GL046268. [Link]

Basu, S., S. Basu, C. E. Valladares, H. C. Yeh, S. Y. Su, E. MacKenzie, P. J. Sultan, J. Aarons, F. J. Rich, P. Doherty, K. M. Groves, and T. W. Bullett, 2001: Ionospheric effects of major magnetic storms during the international space weather period of September and October 1999: GPS observations, VHF/UHF scintillations, and in situ density structures at middle and equatorial latitudes. J. Geophys. Res., 106, 30389-30413, doi: 10.1029/2001JA001116. [Link]

Burnside, R. G., J. W. Meriwether Jr., and M. R. Torr, 1977: Contamination of ground-based measurements of OI

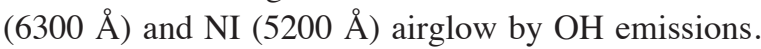
Planet. Space Sci., 25, 985-988, doi: 10.1016/0032-06
33(77)90012-5. [Link]

Burnside, R. G., F. A. Herrero, J. W. Meriwether Jr., and J. C. G. Walker, 1981: Optical observations of thermospheric dynamics at Arecibo. J. Geophys. Res., 86, 5532-5540, doi: 10.1029/JA086iA07p05532. [Link]

Chen, A. B.-C., Y. J. Wu, C. Y. Chiang, Y. C. Huang, C. L. Kuo, H. T. Su, R. R. Hsu, S. B. Mende, H. U. Frey, S. E. Harris, Y. Takahashi, and L. C. Lee, 2012: Sensitivity degradation of ISUAL instruments and its impact on observations. Terr. Atmos. Ocean. Sci., 23, 71-83, doi: 10.3319/TAO.2011.06.20.01(AA). [Link]

Chern, J. L., R. R. Hsu, H. T. Su, S. B. Mende, H. Fukunishi, Y. Takahashi, and L. C. Lee, 2003: Global survey of upper atmospheric transient luminous events on the ROCSAT-2 satellite. J. Atmos. Sol.-Terr. Phys., 65, 647-659, doi: 10.1016/S1364-6826(02)00317-6. [Link]

Colerico, M. J. and M. Mendillo, 2002: The current state of investigations regarding the thermospheric midnight temperature maximum (MTM). J. Atmos. Sol.-Terr. Phys., 64, 1361-1369, doi: 10.1016/S1364-6826(02)00 099-8. [Link]

Colerico, M. J., M. Mendillo, D. Nottingham, J. Baumgardner, J. Meriwether, J. Mirick, B. W. Reinisch, J. L. Scali, C. G. Fesen, and M. A. Biondi, 1996: Coordinated measurements of $\mathrm{F}$ region dynamics related to the thermospheric midnight temperature maximum. J. Geophys. Res., 101, 26783-26793, doi: 10.1029/96 JA02337. [Link]

Harper,R.M., 1973: Nighttime meridional neutral winds near $350 \mathrm{~km}$ at low to mid-latitudes. J. Atmos. Terr. Phys., 35, 2023-2034, doi: 10.1016/0021-9169(73)90116-5. [Link]

Herrero, F. A. and J. W. Meriwether Jr., 1980: 6300- $\AA$ airglow meridional intensity gradients. J. Geophys. Res., 85, 4191-4204, doi: 10.1029/JA085iA08p04191. [Link]

Herrero, F. A. and N. W. Spencer, 1982: On the horizontal distribution of the equatorial thermospheric midnight temperature maximum and its seasonal variation. Geophys. Res. Lett., 9, 1179-1182, doi: 10.1029/GL009i01 0p01179. [Link]

Link, R. and L. L. Cogger, 1988: A reexamination of the O I 6300-Å nightglow. J. Geophys. Res., 93, 9883-9892, doi: 10.1029/JA093iA09p09883. [Link]

Maruyama, T., S. Saito, M. Kawamura, and K. Nozaki, 2008: Thermospheric meridional winds as deduced from ionosonde chain at low and equatorial latitudes and their connection with midnight temperature maximum. J. Geophys. Res., 113, A09316, doi: 10.1029/20 08JA013031. [Link]

Mende, S. B., G. R. Swenson, S. P. Geller, R. A. Viereck, E. Murad, and C. P. Pike, 1993: Limb view spectrum of the Earth's airglow. J. Geophys. Res., 98, 1911719125, doi: 10.1029/93JA02282. [Link] 
Meriwether, J., M. Faivre, C. Fesen, P. Sherwood, and O. Veliz, 2008: New results on equatorial thermospheric winds and the midnight temperature maximum. Ann. Geophys., 26, 447-466, doi: 10.5194/angeo-26-447-20 08. [Link]

Niranjan, K., P. S. Brahmanandam, and B. Srivani, 2006: Signatures of equatorial midnight temperature maximum as observed from in situ and ground-based ionospheric measurements in the Indian sector. J. Geophys. Res., 111, A07309, doi: 10.1029/2005JA011386. [Link]

Otsuka, Y., T. Kadota, K. Shiokawa, T. Ogawa, S. Kawamura, S. Fukao, and S.-R. Zhang, 2003: Optical and radio measurements of a 630-nm airglow enhancement over Japan on 9 September 1999. J. Geophys. Res., 108, 1252, doi: 10.1029/2002JA009594. [Link]

Otsuka, Y., K. Shiokawa, T. Ogawa, and P. Wilkinson, 2004: Geomagnetic conjugate observations of medium-scale traveling ionospheric disturbances at midlatitude using all-sky airglow imagers. Geophys. Res. Lett., 31, doi: 10.1029/2004GL020262. [Link]

Peterson, V. L., T. E. VanZandt, and R. B. Norton, 1966: Fregion nightglow emissions of atomic oxygen: 1 . Theory. J. Geophys. Res., 71, 2255-2265, doi: 10.1029/JZ 071i009p02255. [Link]

Pimenta, A. A., Y. Sahai, J. A. Bittencourt, and F. J. Rich, 2007: Ionospheric plasma blobs observed by OI 630 $\mathrm{nm}$ all-sky imaging in the Brazilian tropical sector during the major geomagnetic storm of April 6-7, 2000. Geophys. Res. Lett., 34, L02820, doi: 10.1029/2006G L028529. [Link]

Rajesh, P. K., J. Y. Liu, C. Y. Chiang, A. B. Chen, W. S. Chen, H. T. Su, R. R. Hsu, C. H. Lin, M.-L. Hsu, J. H. Yee, and J. B. Nee, 2009: First results of the limb imaging of $630.0 \mathrm{~nm}$ airglow using FORMOSAT-2/Imager of Sprites and Upper Atmospheric Lightnings. $J$. Geophys. Res., 114, A10302, doi: 10.1029/2009JA0
14087. [Link]

Rishbeth, H., 1972: Thermospheric winds and the $F$-region: A review. J. Atmos. Terr. Phys., 34, 1-47, doi: 10.10 16/0021-9169(72)90003-7. [Link]

Rishbeth, H. and C. S. G. K. Setty, 1961: The F-layer at sunrise. J. Atmos. Terr. Phys., 20, 263-276, doi: 10.10 16/0021-9169(61)90205-7. [Link]

Rishbeth, H. and O. K. Garriot, 1969: Introduction to Ionospheric Physics, Elsevier, Academic Press, New York.

Saito, A., M. Nishimura, M. Yamamoto, S. Fukao, M. Kubota, K. Shiokawa, Y. Otsuka, T. Tsugawa, T. Ogawa, M. Ishii, T. Sakanoi, and S. Miyazaki, 2001: Traveling ionospheric disturbances detected in the FRONT campaign. Geophys. Res. Lett., 28, 689-692, doi: 10. 1029/2000GL011884. [Link]

Shiokawa, K., Y. Otsuka, and T. Ogawa, 2006: Quasiperiodic southward moving waves in 630-nm airglow images in the equatorial thermosphere. J. Geophys. Res., 111, A06301, doi: 10.1029/2005JA011406. [Link]

Spencer, N. W., C. R. Carignan, H. G. Mayr, H. B. Niemann, R. F. Theis, and L. E. Wharton, 1979: The midnight temperature maximum in the Earth's equatorial thermosphere. Geophys. Res. Lett., 6, 444-446, doi: 10.1029/GL006i006p00444. [Link]

Titheridge, J. E. and M. J. Buonsanto, 1983: Annual variations in the electron content and height of the $\mathrm{F}$ layer in the northern and southern hemispheres, related to neutral composition. J. Atmos. Terr. Phys., 45, 683-696, doi: 10.1016/S0021-9169(83)80027-0. [Link]

Torr, M. R. and D. G. Torr, 1973: The seasonal behaviour of the F2-layer of the ionosphere. J. Atmos. Terr. Phys., 35, 2237-2251, doi: 10.1016/0021-9169(73)90140-2. [Link]

Yonezawa, T., 1959: On the seasonal and non-seasonal annual variations and the semi-annual variation in the noon and midnight densities of the F2-layer in middle latitudes, II. J. Radio Res., 6, 651-660. 